\title{
CP Violation in Strange Baryon Decays: A Report from Fermilab Experiment 871
}

\author{
C. James, ${ }^{a}$ R. A. Burnstein, ${ }^{e}$ A. Chakravorty, ${ }^{e}$ A. Chan, ${ }^{b}$
} Y. C. Chen,${ }^{b}$ W. S. Choong,${ }^{c}$ K. Clark,${ }^{i}$ E. C. Dukes,${ }^{j}$ C. Durandet,${ }^{j}$ J. Felix ${ }^{d}{ }^{\text {R. Fuzesy }},{ }^{c}$ G. Gidal, ${ }^{c}$ H. R. Gustafson, ${ }^{g}$ C. Ho, ${ }^{b}$ T. Holmstrom,${ }^{j}$ M. Huang, ${ }^{j}$ M. Jenkins, ${ }^{i}$ D. M. Kaplan, ${ }^{e}$ L. M. Lederman, ${ }^{e}$ N. Leros,${ }^{f}$ M. J. Longo, ${ }^{g}$ F. Lopez,${ }^{g} \mathrm{~W}$. Luebke, ${ }^{e}$ K. B. Luk ${ }^{c}$ G. Moreno, ${ }^{d}$ K. Nelson ${ }^{j}$ V. Papavassiliou, ${ }^{h}$ J. P. Perroud,${ }^{f}$ D. Rajaram,${ }^{e}$ H. A. Rubin, ${ }^{e}$ M. Sosa,${ }^{d}$ P. K. Teng, ${ }^{b}$ B. Turko, ${ }^{c}$ J. Volk ${ }^{a}$ C. G. White, ${ }^{e}$ S. L. White, ${ }^{e}$ and P. Zyla ${ }^{c}$

a Fermi National Accelerator Laboratory, Batavia, Illinois 60510

${ }^{b}$ Academia Sinica, Nankang, Taipei 11529, Taiwan, R.O.C.

${ }^{c}$ University of California and Lawrence Berkeley Laboratory, Berkeley, California 94720

${ }^{d}$ University of Guanajuato, 37000 Leon, Mexico

e Illinois Institute of Technology, Chicago, IL 60616

${ }^{f}$ University of Lausanne, CH-1015 Lausanne, Switzerland

${ }^{g}$ University of Michigan, Ann Arbor, Michigan 48109

${ }^{h}$ New Mexico State University, Las Cruces, New Mexico, 88003

${ }^{i}$ University of South Alabama, Mobile, Alabama 36688

${ }^{j}$ University of Virginia, Charlottesville, Virginia 22901

\begin{abstract}
Fermilab experiment 871, HyperCP, is a search for direct CP violation in $\Xi$ and $\Lambda$ hyperon decays. A non-zero value in the asymmetry parameter $\mathcal{A}$, defined in terms of the decay parameter products $\alpha_{\Xi} \alpha_{\Lambda}$ and $\alpha_{\Xi} \alpha_{\bar{\Lambda}}$, would be unambiguous evidence for direct CP violation. The first data-taking run finished at the end of 1997 and accumulated over one billion $\Xi^{-}$and $\bar{\Xi}^{+}$decays. A sensitivity in $\mathcal{A}$ of $\approx 10^{-4}$ is expected. A review of CP violation in hyperon decays is given, the HyperCP detector is described, and the status of the data analysis is discussed.
\end{abstract}

\section{INTRODUCTION}

Using the hyperon system to look for evidence of $\mathrm{T}$ or $\mathrm{CP}$ symmetry violation is not a new idea. In fact it is an idea that has been around some 40 years. In the late $1950 \mathrm{~s}$, parity non-conservation was discovered [1] and the impact on hyperon decays was developed and shown [2]. An idea to look for CP violation

CP459, Heavy Quarks at Fixed Target

edited by Harry W. K. Cheung and Joel N. Butler

(C) 1999 The American Institute of Physics 1-56396-864-9/99/ $\$ 15.00$ 
in hyperon decays [3] led to a few experimental searches after CP violation was discovered in $K^{0}$ decays in the early 1960s [4]. Practical experimental efforts were hampered, however, by statistics: making quantities of hyperons was difficult, given the accelerators available in the 1960s and 1970s, and given the hyperon production cross section at those accelerator energies. The high statistics are required to get to the level of CP-odd effects predicted by most models. Within the past 15 years, there have been three experiments which searched for $\mathrm{CP}$ violation in $\Lambda$ decay [5-7]. But none were designed specifically for studying $\mathrm{CP}$ effects, and the results are statistics limited. With the current generation of proton accelerators, along with recent improvements in detector hardware, it is possible to collect large numbers of hyperons in a reasonably short time. HyperCP is the first dedicated hyperon $\mathrm{CP}$ violation experiment.

\section{PHENOMENOLOGY}

All hyperons, spin- $\frac{1}{2}$ strange baryons, decay into a spin- $\frac{1}{2}$ baryon and a pion, reducing strangeness by $1^{1}$. Both $S$ - and $P$-wave final states are allowed by conservation of angular momentum, and only the $P$-wave final state is allowed by conservation of parity. But parity is not conserved in these weak decays, so the decay goes into a mixture of $S$ - and $P$-wave final states. Hyperon non-leptonic decays are conventionally described by the Lee-Yang variables, defined in terms of these $S$ - and $P$-wave amplitudes [8].

$$
\begin{aligned}
& \alpha=\frac{2 R e\left(S^{*} P\right)}{|S|^{2}+|P|^{2}} \\
& \beta=\frac{2 \operatorname{Im}\left(S^{*} P\right)}{|S|^{2}+|P|^{2}} \\
& \gamma=\frac{|S|^{2}-|P|^{2}}{|S|^{2}+|P|^{2}},
\end{aligned}
$$

where the decay parameters $\alpha^{2}+\beta^{2}+\gamma^{2}=1$. The parity violation in the decays shows up as a forward-backward asymmetry in the distribution of the daughters, quantified by the parameter $\alpha$ :

$$
\frac{d N}{d \Omega}=\frac{1}{4 \pi}\left(1+\alpha \vec{P}_{p} \cdot \hat{p}_{d}\right)
$$

where $\vec{P}_{p}$ is the parent hyperon polarization, and $\frac{d N}{d \Omega}$ and $\hat{p}_{d}$ are the daughter baryon distribution and the daughter baryon momentum unit vector, both in the parent's rest frame. The polarization of the daughter baryon is in general given by:

1) Except the $\Sigma^{0}$, which decays electromagnetically into $\Lambda-\gamma$ with $\Delta S=0$. 


$$
\vec{P}_{d}=\frac{\left(\alpha+\vec{P}_{p} \cdot \hat{p}_{d}\right) \hat{p}_{d}+\beta\left(\vec{P}_{p} \times \hat{p}_{d}\right)+\gamma\left(\hat{p}_{d} \times\left[\vec{P}_{p} \times \hat{p}_{d}\right]\right)}{1+\alpha \vec{P}_{p} \cdot \hat{p}_{d}},
$$

which simplifies to

$$
\vec{P}_{d}=\alpha \hat{p}_{d}
$$

if the parent is unpolarized.

Under a CP operation, a daughter baryon emitted in the forward direction (as defined by the parent polarization vector) is transformed into a daughter antibaryon emitted in the backward direction. So if CP is conserved, $\alpha=-\bar{\alpha}$, and, following a similar argument, $\beta=-\bar{\beta}$. Differences in the magnitude of either $\alpha$ or $\beta$ for a particle-antiparticle pair indicate direct CP violation.

In practice, experimental measurements of $\beta$ to the necessary precision are difficult, requiring the measurement of both the daughter and parent polarization. Measurement of $\alpha$ can be made simple by utilizing a feature of hyperon production dynamics. In a fixed target experiment using protons, $\Xi$ s are produced through the reaction $p+$ Nucleon $\rightarrow \Xi^{ \pm}+X$. A $\Xi$ emerging with $p_{t} \cong 0$ is unpolarized due to parity conservation in the strong interaction which produced it. The $\Xi$ decays to a $\Lambda$ and $\pi$, with, following equation (6), the $\Lambda$ in a helicity state with known polarization:

$$
\vec{P}_{\Lambda}=\alpha \equiv \hat{p}_{\Lambda} .
$$

The $\Lambda$ decays in turn to a proton and $\pi$, with the proton distribution given by equation (4), which is rewritten as

$$
\frac{d N}{d \cos \theta}=\frac{1}{2}\left(1+\alpha_{\Lambda} \alpha_{\Xi} \cos \theta\right)
$$

where $\theta$ is the angle between the proton momentum and $\Lambda$ polarization vectors in the $\Lambda$ rest frame. The decay parameter product $\alpha_{\Xi} \alpha_{\Lambda}$ is extracted by measuring the slope of the decay angle $\theta$ distribution. $\mathrm{A} \mathrm{CP}$ observable $\mathcal{A}$ is defined as

$$
\mathcal{A}=\frac{\alpha_{\Xi} \alpha_{\Lambda}-\alpha_{\Xi} \alpha_{\bar{\Lambda}}}{\alpha_{\equiv} \alpha_{\Lambda}+\alpha_{\Xi} \alpha_{\bar{\Lambda}}} \simeq A_{\Xi}+A_{\Lambda} .
$$

$\mathcal{A}$ is determined by measurement of $\alpha_{\Xi} \alpha_{\Lambda}$ from $\Xi^{-}$decays and $\alpha_{\Xi} \alpha_{\bar{\Lambda}}$ from $\bar{\Xi}^{+}$ decays. Using this method, one cannot distinguish whether an observed asymmetry originates in the decay of the $\Xi$ or the $\Lambda$, since $\alpha_{\Xi}$ and $\alpha_{\Lambda}$ are not measured separately.

Calculating $A_{\Xi}$ and $A_{\Lambda}$ is difficult and model dependent, requiring knowledge of poorly understood hadronic matrix elements [9]. Published predictions for $A_{\Xi}$ and $A_{\Lambda}$ range in magnitude from $1 \times 10^{-5}$ to $6 \times 10^{-4}$ [10-15]. Models with no $\Delta \mathrm{S}=1 \mathrm{CP}$-odd effects, such as the superweak model of Wolfenstein [16], predict no asymmetry. 


\section{THE $H Y P E R C P$ EXPERIMENT}

$\mathrm{CP}$ violation in hyperon decay is best studied using a simple, high rate spectrometer. High rate is required to achieve the required statistics, while simplicity is demanded to keep systematic effects small and controllable. The HyperCP spectrometer is shown schematically in Figure 1.

\section{The Spectrometer}

Es are produced by steering an $800 \mathrm{GeV}$ proton beam into a $2 \mathrm{~mm}$ square target. Immediately following the target is a curved collimator imbedded in a $6 \mathrm{~m}$ long 1.6-Tesla dipole magnet. The collimator serves the dual purpose of selecting a narrow momentum range of charged particles while stopping all primary protons which did not interact in the target. The mean momentum selected is about 160 $\mathrm{GeV} / \mathrm{c}$, at which the $\Xi$ to charged particle ratio is approximately maximal. The charge of the secondary beam is selected with the sign of the dipole field; $\Xi^{-}$and $\bar{\Xi}^{+}$decays cannot be collected simultaneously. A typical primary beam intensity of $1.5 \times 10^{11}$ protons per $20 \mathrm{~s}$ spill gives a secondary beam rate of $20 \mathrm{MHz}$, dominated by protons, pions, and kaons. By aiming the primary protons at 0 degrees wrt the long axis of the collimator, the $\Xi$ accepted through the channel will have zero polarization.
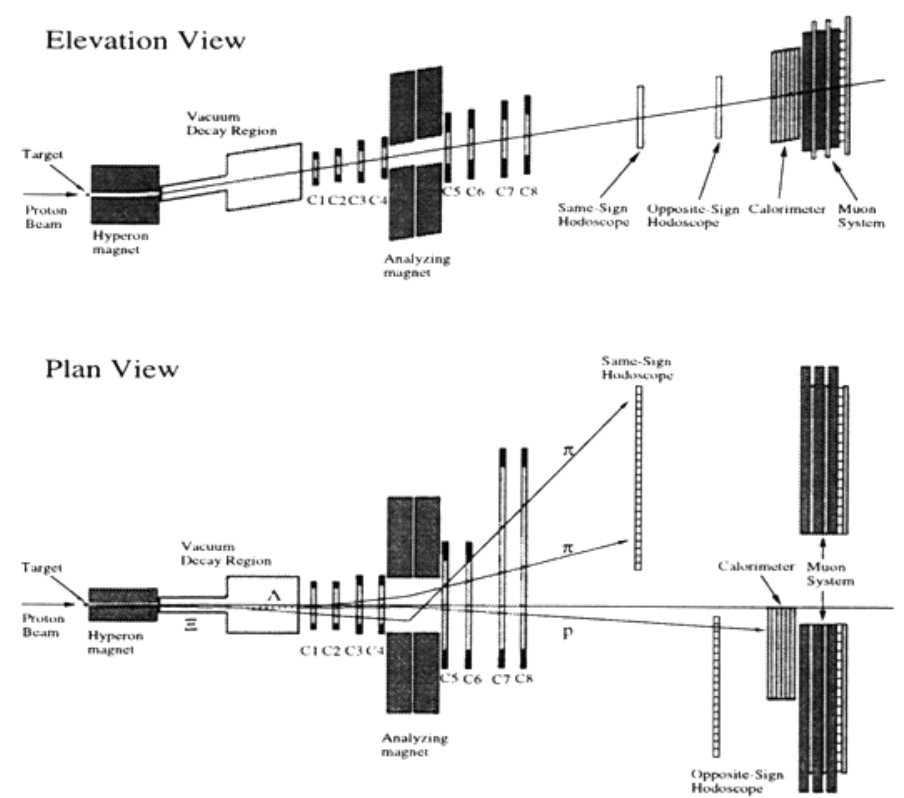

FIGURE 1. Schematic diagram for the Hyper $C P$ spectrometer. 
Upon exiting the collimator, the secondary beam traverses a 13 meter evacuated decay region where most of the hyperons decay. After the decay region, the beam enters the spectrometer proper, made from high rate, narrow pitch proportional wire chambers. The analyzing magnet has sufficient strength to insure that the proton from the $\Lambda$ decay is always bent to one side (the 'opposite sign' side) and the two pions from the $\Xi$ and $\Lambda$ decays are always bent to the other ('same sign') side, and both are well separated from the intense secondary beam. Farther downstream are two scintillation hodoscopes, a hadronic calorimeter used as a trigger element, and a muon detection station. Helium bags are positioned between all the detector components to reduce multiple scattering and secondary interactions.

The $\Xi$ trigger simply requires a coincidence of at least 1 hit in each of the hodoscopes, along with a calorimeter energy signature; the calorimeter threshold is set not only to exclude muons, but also to reduce triggers caused by low energy hadrons produced from beam interactions in the spectrometer material. By reversing the polarities of both the collimator and analysis magnets, $\Xi^{-}$and $\bar{\Xi}^{+}$decays are collected with the identical trigger. A typical $\Xi$ trigger rate is $30 \mathrm{kHz}$. Additional triggers are used to select kaon and muon events, for detector monitoring and systematics studies. A typical overall trigger rate is $75 \mathrm{kHz}$.

The sheer size of the data set to be recorded mandates a highly capable data acquisition system. Simplicity and parallelism in the readout and DAQ are the hallmarks [17]. Average dead time per event is $3 \mu \mathrm{s}$. The average event size during standard running is 550 bytes. With the trigger rate listed above, HyperCP produces data at $41 \mathrm{MB} / \mathrm{sec}$ during spill. Spill is delivered 19 seconds every minute for an average data to tape rate of $13 \mathrm{MB} / \mathrm{sec}$. The maximum sustainable data rate to tape achieved by Hyper $C P$ is $17 \mathrm{MB} / \mathrm{sec}$ (a world record for a HEP experiment). Despite the high rate of the secondary beam and trigger, most events reconstruct cleanly, as shown in Figure 2.

\section{Status of the Analysis}

The 1996-1997 fixed target program at FNAL came to an end on September 5th 1997. By that time, Hyper $C P$ had written to tape 75 billion triggers of all types. 39 billion $\Xi$ triggers have been recorded on 38 TB of storage. Preliminary analysis of $\sim 10$ percent of the data indicates that we have accumulated 1.2 billion $\Xi^{-} \rightarrow \Lambda \pi^{-}$ decays and 0.3 billion $\Xi^{+} \rightarrow \bar{\Lambda} \pi^{+}$decays. 280 million $K^{ \pm} \rightarrow 3 \pi$ decays, as well as a large number of $\Omega \rightarrow \Lambda K$ decays were also collected. The estimated statistical uncertainty for the $\mathrm{CP}$ violation observable $\mathcal{A}$ with this data set is $\sim 2 \times 10^{-4}$. Additional smaller data sets are available with different target materials $(\mathrm{Cu}, \mathrm{W}$, and $\mathrm{Be}$ ) to study $A$ dependence in hyperon yield, and with non-zero production angles to study polarized hyperons. Event reconstruction of the full data set is currently underway. A preliminary CP measurement is not yet available. However, to give an idea of the quality of the data, Figure 3 shows the reconstructed $\Lambda \pi$ invariant masses from about 20 million $\Xi$ events of both polarities. The mass 
resolution is about $1.6 \mathrm{GeV} / \mathrm{c}^{2}$. There is good agreement between the $\Xi^{-}$and $\Xi^{+}$ distributions, and low background.

\section{Systematics}

A precision measurement can only be successful if systematic effects can be controlled and understood. In general, the potential sources of bias are: acceptance differences between $\Xi^{-}$and $\Xi^{+}$decays, non-zero polarization of the parent $\Xi$, and differences in the particle anti-particle interaction rate within the spectrometer (i.e. $p$ versus $\bar{p}$ interaction cross sections), and differences in the backgrounds under the $\Xi^{-}$and $\bar{\Xi}^{+}$and the $\Lambda$ and $\tilde{\Lambda}$ mass distributions.

Because the $\alpha$ parameters for the $\Xi$ and $\Lambda$ both change sign under CP, the proton decay distribution in the $\Lambda$ rest frame should be identical to the anti-proton decay distribution in the $\bar{\Lambda}$ rest frame (assuming the parent $\Xi$ s are unpolarized and $\mathrm{CP}$ is conserved). One might think no acceptance corrections are required; however the production mechanisms and therefore the momentum spectra of $\Xi^{-}$and $\bar{\Xi}^{+}$are different, so at least that correction is required. Implicit in the goal to minimize other acceptance corrections is that the magnetic fields, detector components, and reconstruction efficiencies remain stable between positive running ( $\bar{\Xi}^{+}$data) and negative running ( $\Xi^{-}$data). To facilitate stability, the running polarity is changed frequently (about every 4 hours at full beam intensity), the magnetic fields are monitored with high precision Hall probes, and a uniform secondary beam flux is

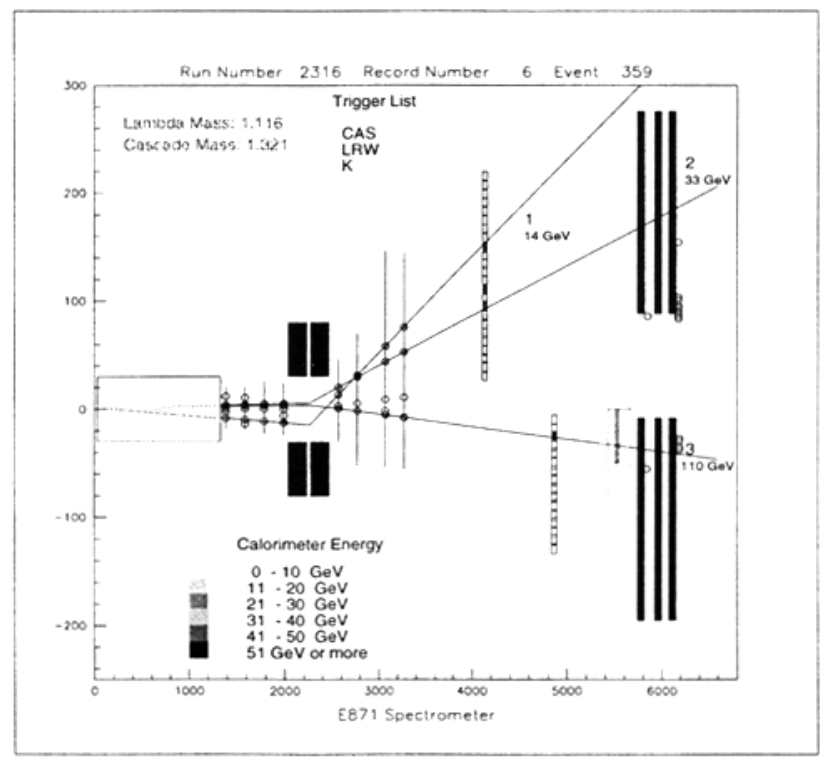

FIGURE 2. Event display for a reconstructed $\Xi$ decay. 
maintained by using different length targets for the two beam polarities. Given that real experiments are never ideal, acceptance variations will be tracked and corrected as the data analysis proceeds.

The $\Xi \rightarrow \Lambda \pi ; \Lambda \rightarrow p \pi$ decay topology naturally reduces systematic acceptance errors because the $\Lambda$ polarization vector is not fixed with respect to the lab coordinates. In the $\Xi$ rest frame, the $\Lambda$ decays throughout the $4 \pi$ solid angle. Each value of $\theta$ in equation (8), therefore, probes a large geometric region within the spectrometer, such that local chamber inefficiencies and geometric acceptance variations map across a wide range of $\cos \theta$. This effect, along with high acceptance for all $\Xi$ decays, significantly dilutes potential biases due to acceptance differences.

Due to mistargeting and the finite size of the hyperon channel, some parent $\Xi \mathrm{s}$ may be produced with non-zero polarization. The magnitude of this polarization, although expected to be quite small, will be measured and any bias can, in principle, be removed (if required). For these events, the parent $\Xi$ will have a small, fixed polarization in the lab frame; however, in the $\Lambda$ rest frame, the effects of this polarization will be diluted as discussed above.

A real-life estimate of experimental errors using differing samples of $\Xi^{-}$decays confirms the ruggedness of this method. Pairs of data sets taken with various values of $p_{t}$ and $\vec{p}_{\Xi}$ are compared without making any acceptance corrections. Since only $\Xi^{-}$events are used, all observed difference are due to biases. When the samples differ significantly in mean momentum, biases are observed; however, as these differences diminish (e.g. $\Delta p<20 \mathrm{GeV} / c$ ), no statistical differences in the

\section{Mass comparison}

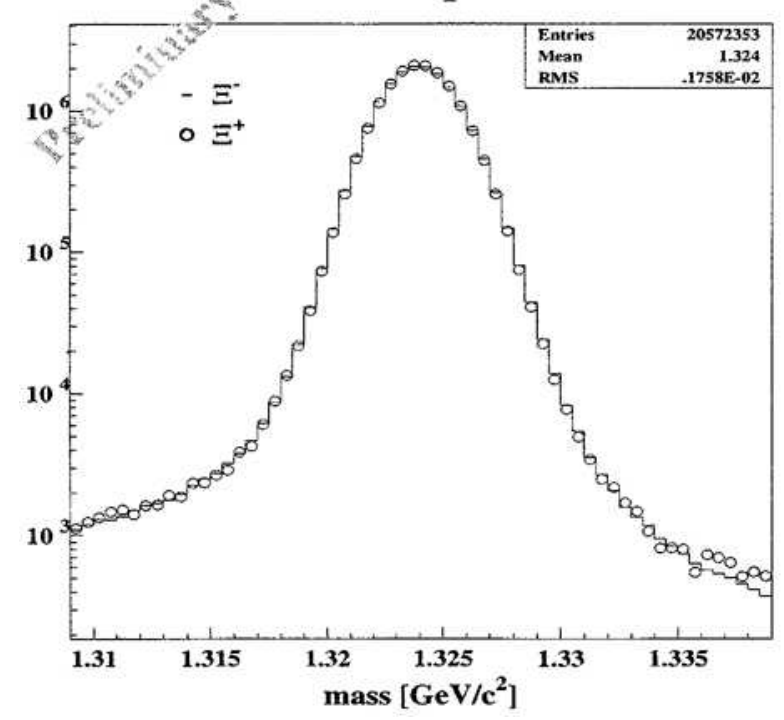

FIGURE 3. The $\Lambda \pi$ invariant mass for positive and negative $\Xi$ trigger events. 
$\cos \theta$ distributions are present down to a few parts per thousand. No statistical differences are found in samples with similar $\Xi$ momentum, yet widely differing polarizations. For example, Figure 4 shows the $\cos \theta$ distributions in the $\Lambda$ helicity frame for two data sets where the $\Xi^{-}$were produced at opposite non-zero production angles, giving them opposite and relatively large polarizations. Studies so far show that without corrections, most biases should not contribute to a false asymmetry in $\mathcal{A}$ above the $10^{-4}$ level.

Finally, differences in particle and anti-particle interaction cross sections are currently being studied. Corrections will be required; however, we expect to understand this bias to $\sim 1 \times 10^{-4}$. Differences in the backgrounds of the reconstructed hyperons and anti-hyperons are small, and are also being studied.

\section{FUTURE PROSPECTS AND ACKNOWLEDGEMENTS}

The HyperCP collaboration is preparing for a second run in 1999. Modest upgrades to the detector and DAQ should enable a four-fold increase in statistics resulting in a sensitivity in $\mathcal{A}$ of better than $1 \times 10^{-4}$.

The Hyper $C P$ collaboration gratefully acknowledges the efforts of the FNAL staff in providing us with a good run in 1997, and for their assistance in preparations for 1999 running. This work was supported in part by the U.S. Dept. of Energy, the National Science Foundation, and the National Science Council of Taiwan, R.O.C.

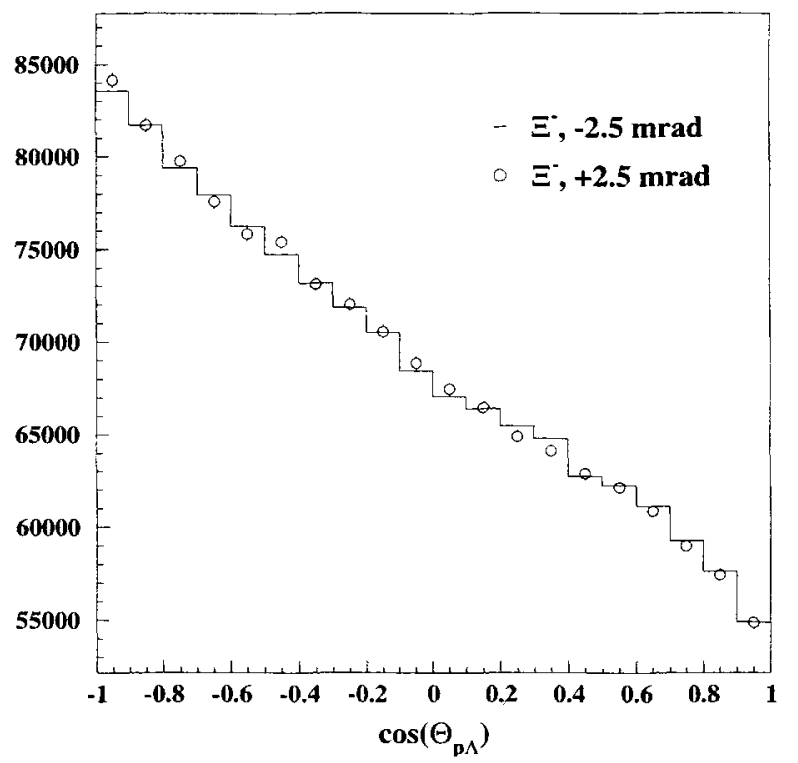

FIGURE 4. The proton $\cos \theta$ distributions in the $\Lambda$ helicity frame for $\Xi^{-}$events from \pm 2.5 mrad production angles. 


\section{REFERENCES}

1. C.S. Wu et al., Phys. Rev. 1051413 (1957).

2. T.D. Lee and C.N. Yang, Phys. Rev. 104254 (1956), F. Crawford et al., Phys. Rev. 1081102 (1957), F. Eisler et al., Phys. Rev. 1081353 (1957), R. Adair and L. Leipuner, Phys. Rev. 1091358 (1958).

3. A. Pais, Phys. Rev. Lett. 3, 242 (1959).

4. J.H. Christenson et al., Phys. Rev. Lett. 13138 (1964).

5. P. Chauvat et al., Phys. Lett. B 163, 273 (1985).

6. M.H. Tixier et al., Phys. Lett. B 212, 523 (1988).

7. P.D. Barnes et al., Phys. Lett. B 199, 147 (1987); P.D. Barnes et al., Phys. Rev. C 54, 1877 (1996).

8. T.D. Lee and C.N. Yang, Phys. Rev. 1081645 (1957).

9. X.G. He and G. Valencia, Phys. Rev. D52, 5257 (1995).

10. J.F. Donoghue, and S. Pakvasa, Phys. Rev. Lett. 55162 (1985).

11. J.F. Donoghue, X.G. He, and S. Pakvasa, Phys. Rev D34 833 (1986).

12. D. Chang, X.G. He, and S. Pakvasa, Phys. Rev. Lett. 743927 (1995).

13. X.G. He, H. Steger, and G. Valencia, Phys. Lett. B272 411 (1991).

14. J.F. Donoghue, Third Conf. on the Intersections between Particle and Nuclear Physics, Rockport ME, May 1988.

15. X.G. He, Proceedings of DPF94, Albuquerque, New Mexico, August 1994.

16. L. Wolfenstein, Phys. Rev. Lett. 13, 562 (1964).

17. D.M. Kaplan et al., Sixth Annual LeCroy Conference on Electronics for Particle Physics, Chestnut Ridge, NY, May 28-29, 1997. 